\title{
Revisiting Character-Based Affective Storytelling under a Narrative BDI Framework
}

\author{
Federico Peinado ${ }^{1}$, Marc Cavazza² and David Pizzi ${ }^{2}$ \\ ${ }^{1}$ Facultad de Informática, Universidad Complutense de Madrid, Spain \\ email@federicopeinado.com \\ ${ }^{2}$ School of Computing, University of Teesside, United Kingdom \\ \{m.o.cavazza, d.pizzi\}@tees.ac.uk
}

\begin{abstract}
Belief-Desire-Intention (BDI) is a well-known cognitive theory, especially in the field of Software Agents. Modelling characters using software agents has been proven to be a suitable approach for obtaining emergent and autonomous behaviours in Interactive Storytelling. In this paper it is claimed that an effective extension of previous models to the BDI framework is useful for designing intelligent characters. An example shows how internal thoughts and motivations of Madame Bovary's main characters can be more naturally formalised as a cognitive side of the story. A narrative reformulation of BDI theory is needed to avoid the implicit complexity of other proposals.
\end{abstract}

Keywords: Story Generation, Software Agents, Artificial Intelligence

\section{Introduction}

Character-based approaches to Interactive Storytelling can be inspired on many different theories. A distinction which is particularly interesting in our research field is made between cognitive-oriented and narrative-oriented models.

While some researchers focus on simulations of human behaviour in which Storytelling is only a promising field of application, others are convinced that an excessive effort on modelling the rationale behind characters distracts us from the real problems of narrative phenomena. As revealed by current studies, a trade-off between narrative and cognitive orientations to character-based storytelling is needed in order to build a system capable of complex and deep storytelling with a balanced integration of these aspects. Belief-Desire-Intention (BDI) theory is starting to be applied in the field as a promising framework for that integration.

In this paper we study the use of BDI for modelling intelligent characters and develop our own Narrative BDI extension to an existing character-based storytelling system, in order to determine its real potential as an explanatory framework for the motivations of characters' behaviour.

${ }^{1}$ Funded by University of Teesside under the RIF initiative, Ministerio de Educación y Ciencia (TIN2006-14433-C02-01), Universidad Complutense de Madrid and Dirección General de Universidades e Investigación de la Comunidad de Madrid (CCG07-UCM/TIC 2803). 


\section{Related Work}

The original Bratman's theory of BDI [1] is a psychological model of human practical reasoning, created as an explanation of future-directed intentions in terms of current knowledge and goals of a rational agent. Different BDI architectures [9, 10] have evolved from this theory which bestows three mental attitudes for determining agent's responses to perceived changes in the environment: beliefs, desires and intentions. Beliefs are facts about the world, including the agent itself, which may not necessarily be true. Desires are world states that the agent would like to accomplish, normally described as a set of goals. Intentions are internal states about what the agent is doing for satisfying its desires, usually executing or deliberating plans.

As the original theory suggests and following works explore [5] there is a complementary relationship between BDI and planning. Nowadays many storytelling systems rely on an automatic planner, implementing characters' desires as goals and characters' intentions as plans, which are composed by operators representing the sequence of actions that the character will try to perform following its desires.

\subsection{Social Behaviour over BDI}

Chang and Soo [3] claim that planning about how to influence the minds of others is a key piece to the generation of stories with sophisticated characters, as Bremond's [2] pointed out before. For modelling characters with social behaviour they propose a BDI architecture in combination with an external "social planner". Their agent-based story generator is based on Shakespeare's Othello, which, as it is shown in Figure 1, contains a remarkable example of a manipulative character, Iago.

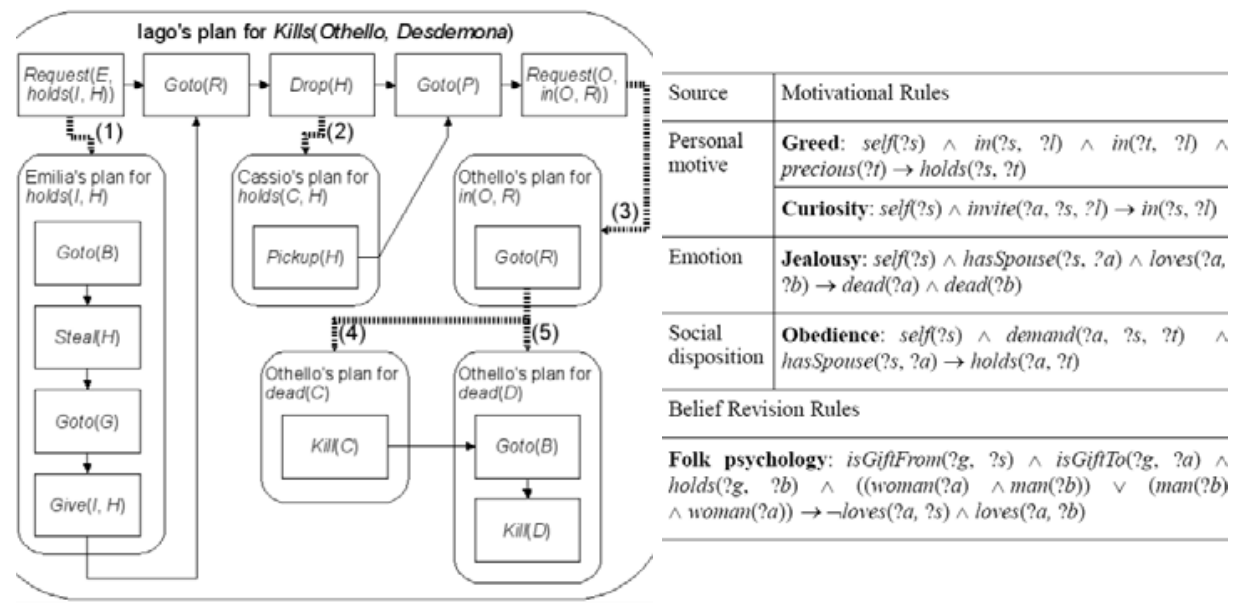

Fig. 1. Sample of the social planning proposal by Chang and Soo [3].

Social plans involve special actions of other agents apart from those than can be performed by their author. These special actions are called foreign actions as opposite to the native actions of the performing agent. Social plans do not use any social- 
specific operators but usual ones as those corresponding to physical actions, e.g. go to Cassio's residence, or communicative acts, e.g. request Emilia to steal Desdemona's handkerchief. What are social-specific in this planning are the behaviour rules of Iago, the social agent. Some of these rules are used for updating agent's beliefs about others according to its perceptions, e.g. if Othello finds a gift that he has given to Desdemona in the Cassio's residence, he will infer she is loving Cassio, not him. Other rules are use for updating agent's goals according to its beliefs about others, e.g. if Othello thinks Desdemona is loving Cassio, he will desire to kill them both.

Foreign actions need their performer to be motivated in some way by the author of the social plan. Preconditions of each foreign action must include the requirement, , that some of its effects match some goals of the performing agent. By-passing the question of agents' perception, effects of both foreign and native actions can cause changes in other characters' beliefs and goals.

\subsection{BDI, Emotions and Drama}

Damiano and Pizzo [4] pointed out the importance of a mature cognitive model of characters' emotions as the ground on top of which the plot is developed. They distinguish between "character as agent" at the cognitive level of actions and "character as drama function" at the narrative level of plot.

In their work, a theoretical view of the BDI framework is enriched with that distinction and the use of emotions, being used to analyse a scene of Shakespeare's Hamlet. As shown in Figure 2, the protagonist is who really drives the scene, while Ophelia simply reacts to his actions.

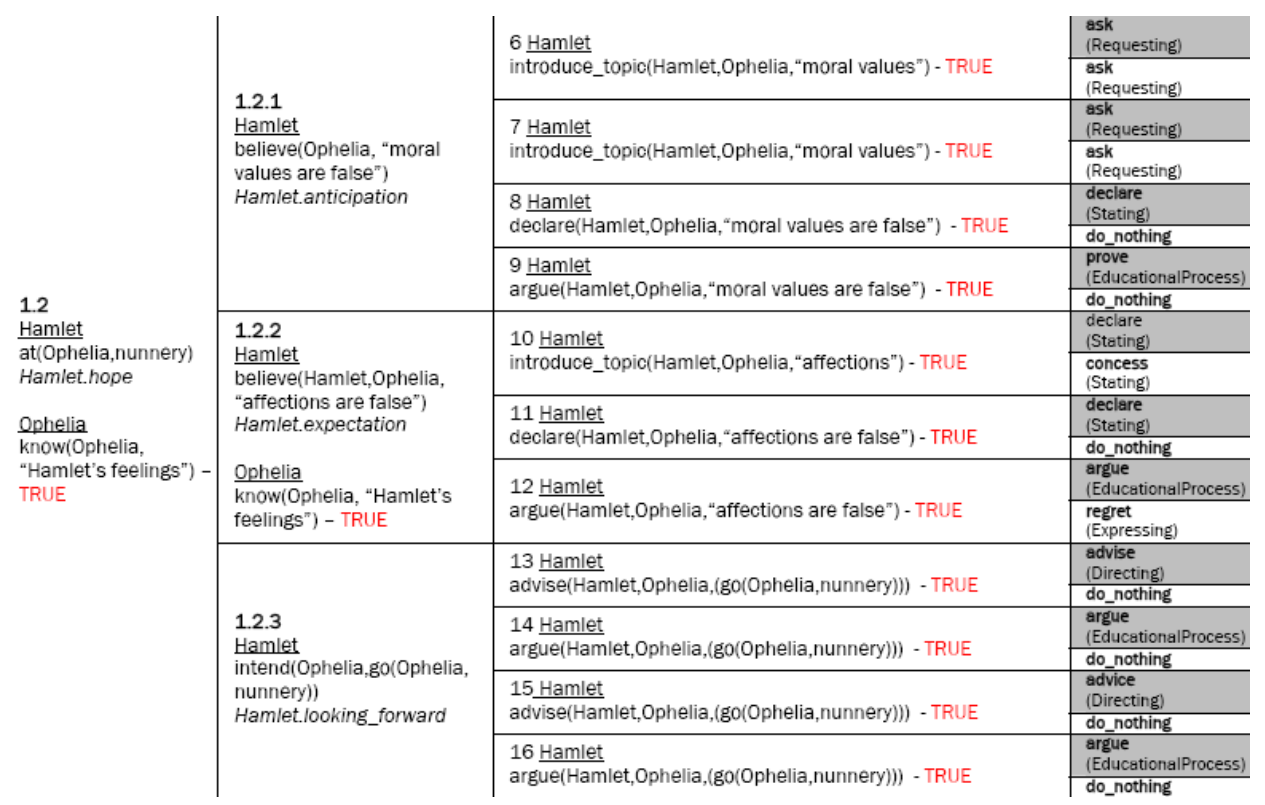

Fig. 2. Sample of the BDI with emotions proposal by Damiano and Pizzo [4]. 
The methodology of these authors has two parts, a bottom-up analysis of the script and a subsequent reformulation of the story according to the proposed model.

The first step of their analysis is to identify the verbal and non-verbal actions at present time; then, drama units along the different layers of the plot tree should be recognised. Drama units have a character as subject and a dramatic goal associated to it which is connected to the frustration or the achievement (i.e. boolean result) of the corresponding agent's physical goal.

Their reformulation starts with the assignment of emotions to the agents, according to their cognitive states, after that, actions are mapped to processes and finally reorganised in a sequence of beats, i.e. action-reaction pairs. Characters are represented as a selection of those beliefs and emotions of their cognitive avatars that are relevant in the context of their dramatic units, i.e. high-level affective conflicts.

\section{The Narrative BDI Model}

This research is a continuation on previous work on Character-Based Affective Storytelling, on top of which our Narrative BDI Model is applied to enrich it with a cognitive layer. The referent is the Interactive Storytelling prototype published by Pizzi et al. [8], in which part of the novel Madame Bovary [6] is formalised.

The approach is based on a real-time planner whose operators mainly deal with literary feelings (as described by Flaubert himself [7]), instead of low-level mental or physical states, so they are called emotional operators. These are classified in three semantic categories. Interpretation operators update characters' feelings to respond to a change in the state of the world. Interaction operators intentionally modify other characters' beliefs. Finally physical operators basically change characters' locations.

The Narrative BDI model is a high-level narrative-oriented extension to the BDI approaches described in Section 2. As in previous approaches, only the relevant characters of a story require formalization as real BDI agents. Beliefs and feelings are directly modified by the effects of perceived emotional operators, the atomic actions of our representation. Desires and goals are usually updated in terms of character's feelings, the atomic facts. Intentions are divided in those that are part of a rational plan and those that are merely reactions. Both are composed only of interaction and physical operators because interpretation operators cannot be triggered by characters. These operators are under the control of the planner, representing that feelings are not usually updated by voluntary acts but according to characters' predefined personality.

The new formalisation of the Madame Bovary sample in terms of the Narrative BDI Model is summarized in Figure 3. Emma experiences the larger repertory of feelings and consequently is the protagonist in most of the operators, but Rodolphe is clearly leading these chapters of the novel from a rational point of view.

In Madame Bovary feelings play a dominant role and characters as Emma present modelling difficulties from the BDI point of view. While Rodolphe is an exemplary instance of a rational agent with pertinent beliefs about women's world, a clear goal and a detailed plan to achieve it, Emma lives a naive fantasy (even believing Rodolphe's lies), her desires are changeable and her behaviour is quite opportunistic. 

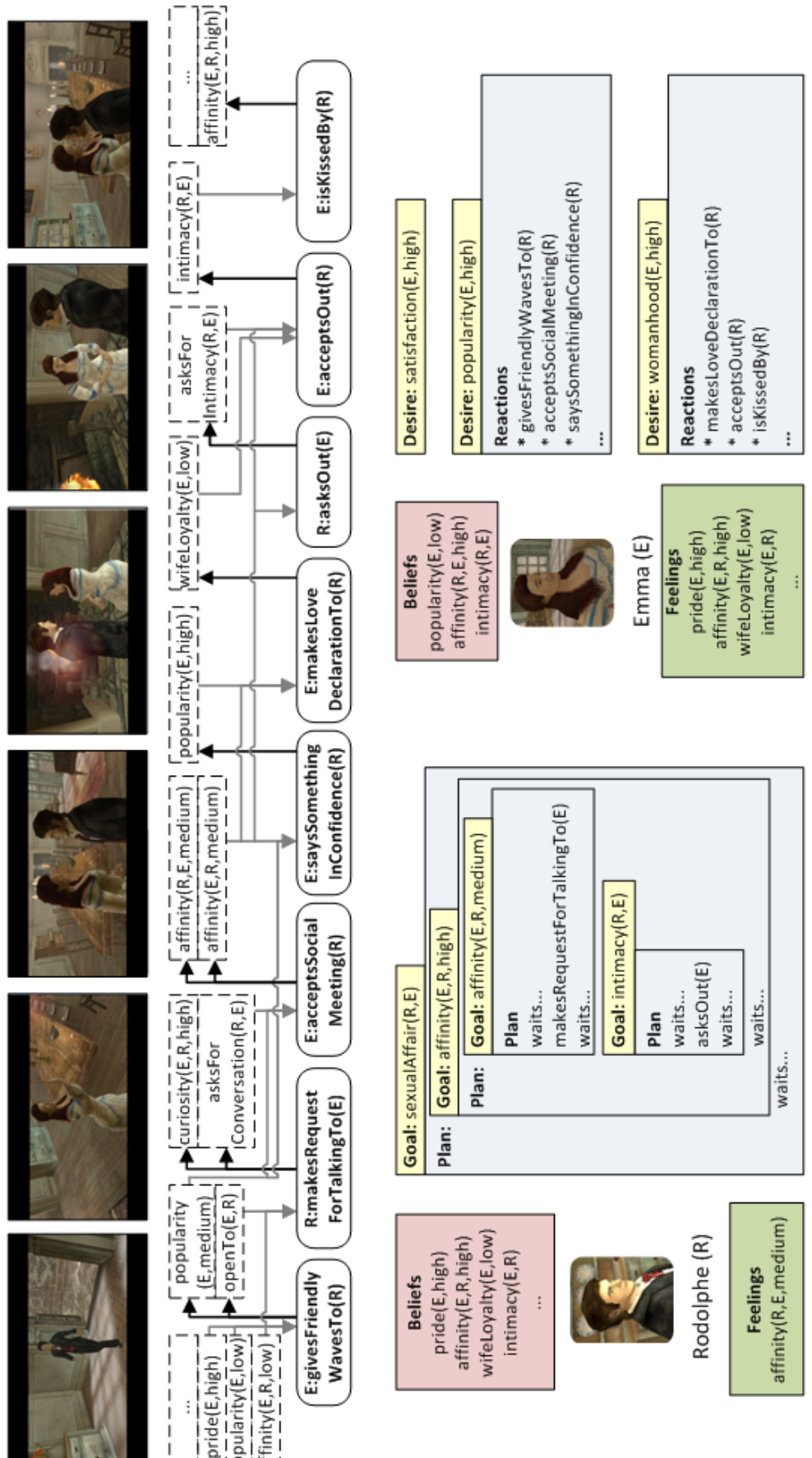

Fig. 3. Mapping the emotional planning approach to the Narrative BDI model. 


\section{Conclusions}

BDI theory is starting to be considered a promising tool for modelling sophisticated characters in Interactive Storytelling. During this theoretical analysis of the framework and its potential benefits for reformalising our character-based storytelling prototypes with motivated characters, four main advantages have been identified.

First, BDI as a cognitive model reinforces narrative causality insofar as motivations and beliefs are causal links that enrich characters. Second, the autonomy of software agents applies a more scalable structure to the way characters' beliefs and feelings are updated: BDI revision rules are distributed among the agents, which is not the case with planning operators. Third, the Narrative BDI model supports characters' behaviour visual explanation, illustrating in a more plausible fashion what is implicit and centralized in the sequence of emotional operators generated by our reused planner. Fourth, although not explored in showed samples, multi-agent systems provide a natural approach to user interaction by simply adding a usercontrolled agent to the repertory.

Most of the benefits derived from Chang and Soo's proposal (stronger social interactions and nested multi-character plots) are consequences of the interaction between many social agents, but as the reader should have notice neither of the three samples gathered here explore stories with more than one "full BDI" agent. Interoperability issues between the functionality provided by BDI platforms and external planners are also an interesting topic for further research.

\section{References}

1. Bratman, M.E.: Intention, Plans, and Practical Reason. CSLI Publications (1999)

2. Bremond, C.: Logique du Recit, Paris. Editions du Seuil (1973) (in French)

3. Chang, P. H-M., Soo, V.-W.: Planning to Influence Other Characters in Agent-Based Narratives. In: Integrating Technologies for Interactive Stories Workshop, International Conference on Intelligent Technologies for Interactive Entertainment, pp. 12-17 (2008)

4. Damiano R., Pizzo A.: Emotions in Drama Characters and Virtual Agents. In: Technical Report Spring Symposium Series. Stanford University, California (2008)

5. de Silva, L., Padgham, L.: Planning as Needed in BDI Systems (extended version of Planning on Demand in BDI Systems). In: International Conference on Automated Planning and Scheduling. Monterey, California (2005)

6. Flaubert, G.: Madame Bovary. La revue de Paris (Ed.), France (1856) (in French)

7. Leclerc, Y (Ed.): Plans et Scenarios de Madame Bovary. CNRS, France (1995) (in French)

8. Pizzi, D., Charles, F., Lugrin J-L., Cavazza, M.: Interactive Storytelling with Literary Feelings. In: Affective Computing and Intelligent Interaction, pp. 630--641. Springer (2007)

9. Rao, A., Georgeff, M.: Modeling Rational Agents within a BDI-Architecture. In: 2nd International Conference on Principles of Knowledge Representation and Reasoning, pp. 473--484. Morgan Kaufmann, San Mateo, CA (1991)

10.Saadi, A., Sahnoun, Z.: Towards Intentional Agents to Manipulate Belief, Desire and Commitment Degrees. In: ACS/IEEE International Conference on Computer Systems and Applications, pp. 515--520. IEEE Digital Library (2006) 